\title{
Specificity and generalization of perceptual learning in low myopia
}

\author{
Clara Casco $^{\mathrm{a}, *}$, Daniela Guzzon ${ }^{\mathrm{b}}$, Monica Moise ${ }^{\mathrm{b}}$, Antonella Vecchies ${ }^{\mathrm{b}}$, Tiziano Testa ${ }^{\mathrm{b}}$ and \\ Andrea Pavan ${ }^{\mathrm{c}}$ \\ ${ }^{a}$ Department of General Psychology, University of Padova, Via Venezia, Padova, Italy \\ ${ }^{\mathrm{b}}$ Neuro.Vis.U.S.Laboratory, University of Padova, Via Belzoni, Padova, Italy \\ ${ }^{c}$ Institut für Psychologie, Universität Regensburg, Universitätsstr., Regensburg, Germany
}

\begin{abstract}
.
Purpose: In this study we investigated in observers with low myopia: (i) the pattern of lateral interactions between stimuli activating early cortical analyzers and its modulation by perceptual learning (PL), and (ii) whether PL transferred to untrained stimuli and tasks and whether it exhibits interocular transfer.

Method: Participants (seven adults with low myopia) performed 12 training sessions. Participants were trained on a contrast detection task of a central Gabor target flanked by two co-oriented and co-aligned high contrast Gabor patches. Target-to-flankers separation along the vertical axis was varied from 2 wavelengths $(\lambda)$ to $8 \lambda$.

Results: The results showed that before PL facilitatory lateral interactions in the myopic eye were reduced in strength, but PL increased contrast sensitivity and improved facilitatory lateral interactions. However, PL did not transfer to different local/global orientations and lower spatial frequencies. On the other hand, PL resulted in an enhancement of the contrast sensitivity function (CSF) and of the uncorrected visual acuity (UCVA) both in the trained and untrained eye.

Conclusions: Such improvements seem to be associated to a modulation of lateral interactions between target and flankers and it is likely to take place at a level in which the inputs from the two eyes converge.
\end{abstract}

Keywords: Low myopia, perceptual learning, lateral interactions, contrast sensitivity, visual acuity

\section{Introduction}

It is well known that performance on perceptual tasks is improved by practice. This practice effect is known as perceptual learning (PL). PL in adult human observers has been shown for several tasks, such as hyper-acuity (McKee and Westheimer, 1978; Poggio, Fahle et al., 1992) phase discrimination in compound gratings (Fiorentini and Berardi, 1981), motion discrimination (Ball and Sekuler, 1982, 1987; Ball, Sekuler, et al., 1983), texture discrimination based on

*Corresponding author: Clara Casco, Department of General Psychology, University of Padova, Via Venezia 8, 35131, Padova, Italy. Tel.: +39049 827 6610; E-mail: clara.casco@unipd.it. simple and combined features differences (Ahissar and Hochstein, 1996; Casco, Campana et al., 2004; Casco and Campana, 1999; Campana and Casco, 2003; Karni and Sagi, 1991), and contrast polarity (Grieco, Casco et al., 2006). The finding that the effect of training on simple visual features was highly specific for location in the visual field (Ahissar and Hochstein, 1996; Karni and Sagi, 1991; Grieco, Casco et al., 2006; Fiorentini and Berardi, 1980), spatial frequency (Fiorentini and Berardi, 1980), contrast polarity (Grieco, Casco et al., 2006), local and global orientation (Ahissar and Hochstein, 1996; Casco and Campana, 1999; Campana and Casco, 2003; Karni and Sagi, 1991; Fiorentini and Berardi, 1980; Casco, Caputo et al., 2001) and in some case even the eye of origin (Karni and Sagi, 1991; 
Fahle 2004; Schoups, Vogels et al., 2001) suggests that neurons or population of neurons are modified at the earliest stage of visual processing. However, other studies reported that practicing in spatial frequency discrimination (Fiorentini and Berardi, 1981) and contrast detection of Gabor patches yields only weak or no improvements (Dosher and Lu, 2005; Sowden, Rose et al., 2002; Mayer, 1983; Dorais and Sagi, 1997; Maehara and Goryo, 2007).

Similarly, the ability to discriminate between two contrast levels of otherwise identical grating patches does not improves with practice (Dorais and Sagi, 1997; Maehara and Goryo, 2007; Adini, Sagi et al., 2002). On the other hand, it has been shown that the addition of high contrast and collinear Gabor flankers enabled learning contrast detection, thus reducing lateral suppression and increasing lateral facilitation by flankers; such modulations are usually obtained with target-to-flankers separations of 2 and 3-4 wavelengths ( $\lambda$ ) (Adini, Sagi et al., 2002; Polat and Sagi, 1993; Polat and Sagi, 1994a, 1994b; Shani and Sagi, 2005; Polat, 2009; Maniglia, Pavan et al., 2011).

These results support the hypothesis that PL strengthens facilitatory interactions and reduces inhibitory interactions between laterally displaced Gabor patches. Indeed, cortical anatomy reveals that long-range horizontal interactions exist in all brain regions, including the visual cortex (Gilbert and Wiesel, 1983; Rockland and Lund, 1983) that are modulated by perceptual learning (Gilbert, Li et al., 2009). Indeed, there is recent psychophysical evidence of training-dependent reduction of low-level inhibitory lateral interactions that weaken a peripheral suppressive effect known as crowding (Maniglia, Pavan et al., 2011). PL also induces faster processing (for example, it increases reading speed) and overcomes the reduction of facilitation caused by background masking (Polat, Schor et al., 2012). The effect of PL is preserved when the contextual flankers are removed, leading to an enhanced contrast sensitivity function (CSF) (Polat, 2009). This suggests that the learning dependent modulation of excitatory and inhibitory connections between neurons improves the response of visual channels selective to spatial frequencies (Adini, Sagi et al., 2002), increasing signal-to-noise ratio in neural activity (Geisler and Albrecht, 1997). In addition, an outstanding study has provided evidence that abnormal neuronal interactions in amblyopia, i.e., reduced facilitation and increased suppression, can be improved by PL (Polat, Ma-Naim et al., 2004). In this study the authors reported a transfer between different categories such as training on contrast detection and improvement of visual acuity. This result raises two important questions:

(i) whether PL improves high level processing that does not involve specificity for basic features

(ii) whether improvement in contrast sensitivity is essential and precedes improvement in visual acuity (e.g., letter recognition task).

The improvement of contrast sensitivity and visual acuity following PL has also been demonstrated both in observers with low myopia (Durrie and McMinn, 2007; Tan and Fong, 2008) and presbyopia (Polat, 2009; Polat, Schor et al., 2012; Durrie and McMinn, 2007). The vision of myopic and presbyopic individuals is blurred without optical correction, and their corrected contrast sensitivity is reduced (Liou and Chiu, 2001). Therefore, in the case of myopia and presbyopia it is possible that repeated practice in an adapted state to blur produces an improvement in contrast sensitivity.

An open question is whether myopia and presbyopia induce abnormal lateral interactions. Indeed, since facilitatory and inhibitory lateral interactions strengthen as a consequence of co-activation of preand post-synaptic units, similarly to a Hebbian learning mechanism, it is possible that non-optimal activation of spatial frequency visual channels in observers with myopia and presbyopia also weaken lateral interactions. A second question concerns the level at which PL operates. The transfer of learning to visual acuity suggests that visual acuity task share a common mechanism operating either at low or high level.

To shed light on these issues we have used a PL paradigm in monocular vision of myopic observers and asked whether:

(i) the pattern of lateral interactions in observers with low myopia differs from observers with normal vision. This should be regarded as maladaptive plasticity; that is, a long-term reduced efficiency of neural communication which limits the capability of representing the details of the input regardless it comes from corrected or uncorrected vision (Webster, Georgeson et al., 2002).

(ii) PL allows overcoming such modified pattern of lateral interactions.

(iii) such possible modulation is specific for the spatial frequency and orientation of the trained stimulus.

(iv) the modulation of lateral interactions by means of PL enhances contrast sensitivity and visual acuity. 
(v) the enhancement of contrast sensitivity and visual acuity is specific for the trained eye.

\section{Methods}

\subsection{Participants}

Myopic participants, all suffering from functional low myopia with relatively late onset during childhood, were seven healthy volunteers (five males and two females), aged 20-25 years. Observers were selected on the basis of optometric evaluation that included: manifest subjective and objective refractions, best spectacle-corrected visual acuity (BSCVA) and uncorrected visual acuity (UCVA) using $4 \mathrm{~m}$ distance Early Treatment Diabetic Retinopathy Study (ETDRS) charts. Table 1 shows the refraction of each observer.

Inclusion criteria were low myopia of cycloplegic spherical equivalence (SE) within the range of $0.75-1.75$ diopters (D) in the worst eye and with astigmatism not exceeding $0.75 \mathrm{D}$ in either eye. Inclusion criteria were also a stable refractive state with no increase beyond $\pm 0.5 \mathrm{D}$ over the previous 6 months, uncorrected visual acuity better than $0.7 \log$ MAR, with no more than $0.3 \log$ MAR difference between the two eyes, and best spectacle-corrected visual acuity better than 0.05 logMAR (ETDRs logMAR charts). Exclusion criteria included ocular condition or cause of reduced visual acuity other than simple myopia and/or astigmatism. The control group consisted of seven normal sighted participants (four males and three females), aged 20-26 years, with uncorrected visual acuity better than $0.05 \log$ MAR. The participants sat in a dark room at a distance of $57 \mathrm{~cm}$ from the screen. Viewing was monocular with the non-dominant eye and without optical correction for both groups. The dominant eye was patched with a black occluder. It

\section{Table 1}

Refraction (in diopters) of each myopic observer for the trained and untrained eye

\begin{tabular}{lcc}
\hline Observer & Trained eye & Untrained eye \\
\hline MV & -1.00 & $-0.75-0.25 \times 90$ \\
AP & -1.25 & -1.75 \\
SE & -1.75 & -1.75 \\
MC & -1.50 & -2.0 \\
MB & -1.25 & -1.25 \\
LB & -0.75 & -0.75 \\
SC & -1.25 & -1.50 \\
\hline
\end{tabular}

should be noted that at the viewing distance of $57 \mathrm{~cm}$, in myopic observers visual acuity is almost corrected to normal.

However, although the visual input is only partially degraded, communication amongst neurons should be affected if myopia produces a long-term plasticity that reduces the strength of interconnections between neurons (Webster, Georgeson et al., 2002).

Informed written consent was obtained from all observers before the study was initiated. The study and protocol conformed to the tenets of the Declaration of Helsinki.

\subsection{Stimuli}

\subsubsection{PL stimuli}

PL stimuli were displayed on a 19-inch LCD Asus monitor with a refresh rate of $60 \mathrm{~Hz}$, and generated using Matlab Psychtoolbox (Brainard, 1997; Pelli, 1997). The screen resolution was $1280 \times 1024$ pixels. The mean luminance was $50 \mathrm{~cd} / \mathrm{m}^{2}$, measured using a Minolta LS-100 photometer. Stimuli were generated using a gamma-corrected lookup table (LUT) to ensure display linearity.

Stimuli were Gabor patches consisting of a cosinusoidal carrier enveloped by a stationary Gaussian. Each Gabor patch was characterized by its wavelength $(\lambda)$, phase $(\varphi)$, and standard deviation $(\sigma)$ of the luminance Gaussian envelope in the $(\mathrm{x}, \mathrm{y})$ space of the image. Formally each Gabor patch can be expressed as follows:

$$
G(x, y)=\cos ((2 \pi / \lambda) x+\varphi) e^{-\left(x^{2}+y^{2}\right) / \sigma^{2}}
$$

Gabors had a spatial frequency of 6 and $12 \mathrm{cpd}$ with $\sigma=\lambda$ and $\varphi=0$ (even symmetric). During the learning sessions a vertical and centrally presented Gabor target was flanked above and below by two high-contrast Gabor patches (0.6 Michelson contrast), originating a configuration of collinear Gabors. Flankers were always vertically oriented and located at various distances from the target: $2 \lambda, 3 \lambda, 4 \lambda$, and $8 \lambda$, corresponding to $0.33,0.49,0.66$ and $1.33 \mathrm{deg}$ for $6 \mathrm{cpd}$, and $0.16,0.25,0.33$ and $0.66 \mathrm{deg}$ for $12 \mathrm{cpd}$ (Fig. 1).

In the learning sessions, the target was presented in only one of two intervals, each lasting $130 \mathrm{~ms}$ and separated by $500 \mathrm{~ms}$. We used a two-interval forced choice task (2IFC) in which the observer was required to choose which of the two temporal intervals con- 

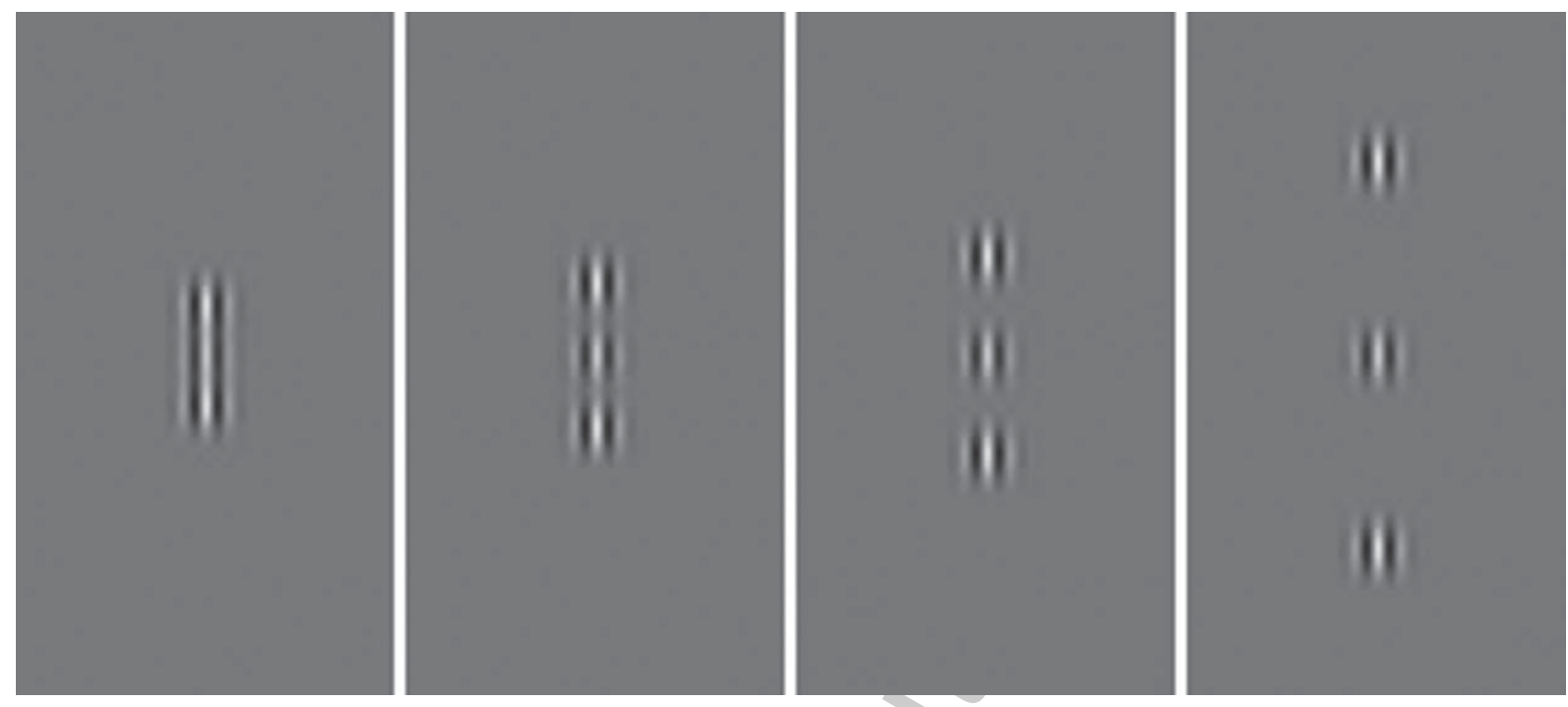

Fig. 1. Stimuli used in the learning sessions. A central target Gabor is flanked by two high-contrast Gabor patches of the same orientation. The figure shows only Gabor patches with a spatial frequency of $6 \mathrm{cpd}$, but in the learning sessions $12 \mathrm{cpd}$ Gabor patches were also employed. Panels from left to right show the four target-to-flankers distances trained: $2 \lambda, 3 \lambda, 4 \lambda$ and $8 \lambda$, respectively. For demonstrative purposes the contrast of the central target Gabor is exaggerated.

tained the target. Contrast detection thresholds were estimated using a 1-up/3-down staircase (Levitt, 1971), with contrast varying in steps of $0.1 \mathrm{log}$ units. The session terminated after either 120 trials or 24 reversals. Threshold was calculated averaging the contrast values in correspondence of the last 18 reversals. Threshold corresponded to the stimulus strength at $79 \%$ accuracy. A fixation point $(0.18 \mathrm{deg})$ indicated the location of the target during the interval between the two presentations. Observers activated the presentation of each pair of images at their own pace. They were informed of a wrong answer by an auditory feedback.

\subsubsection{Baseline PL stimuli}

Lateral interactions and the transfer to other stimulus characteristics were tested by comparing contrast detection thresholds in the pre- and post-test sessions. In the pre- and post-test sessions stimuli were a vertical collinear configuration of vertically oriented Gabor patches and a vertical configuration of Gabor patches with flankers orthogonally oriented with respect to the central target (i.e., orthogonal condition). The spatial frequency was $6 \mathrm{cpd}$ for both groups and $12 \mathrm{cpd}$ only for the myopic observers. Four target-to-flankers distance levels were used: $2 \lambda, 3 \lambda, 4 \lambda$, and $8 \lambda$. In addition, the following conditions were tested: (i) a collinear vertical configuration of $1.5 \mathrm{cpd}$, to test the transfer to a different spatial frequency, (ii) a horizontal collinear configuration of 6 and $12 \mathrm{cpd}$ to test the transfer to a different orientation of the triplet.

\subsubsection{Visual acuity stimuli}

Visual acuity (ETDRs and Landolt-C) was measured before and after PL by using a remote-controlled visual and ophthalmic test chart monitor (Vision Chart by Costruzione Strumenti Oftalmici; CSO) placed at $4 \mathrm{~m}$ of viewing distance on a 19-inch screen with a resolution of $1280 \times 1024$ pixels, contrast of 500:1 and maximum lightness of $280 \mathrm{~cd} / \mathrm{m}^{2}$.

\subsubsection{Eccentric visual acuity and crowding stimuli}

Eccentric visual acuity (eccentric-VA) and crowding were measured before and after PL. Stimuli were generated using E-Prime software and presented at $57 \mathrm{~cm}$ from the same screen used for the PL. The stimuli were 10 SLOAN letters (D, N, S, C, K, R, Z, H, O and V), randomly presented for $100 \mathrm{~ms}$. In the eccentric-VA test, the location of the target letter was 4 deg either to the left or the right (randomly chosen on a trial basis) with respect to the fixation point. The size of the letters varied according to a 1-up/3-down staircase (Levitt, 1971). The step size was 1 font size corresponding to streak width of 0.19 arcmin the character type was Arial, and the starting font size was 20 (streak width of 3.72 arcmin). Observers had to say the letter displayed 
and the experimenter registered the answer. The session terminated after either 100 trials or 18 reversals. The acuity threshold, expressed as the font size for $79 \%$ correct identifications, was the mean of the last eight reversals.

In the crowding test the target letter was flanked on the left and the right sides by two different letters. The triplets could appear randomly either to the left or to the right of the fixation point, but the target letter was always at $4 \mathrm{deg}$ from the fixation spot. In the crowding test, the size of both the target letter and flanking letters was set $30 \%$ bigger than the VA threshold. We measured critical spacing, i.e., inter-letter distance that did not produce threshold elevation, using a 1-up/3-down staircase (Levitt, 1971). The initial distance between letters was set at 95 arcmin. The step size was 5.7 arcmin for the first 5 reversals, and 1.9 arcmin for the remaining reversals. The session terminated either after 100 trials or 18 reversals. At the end of the procedure, we calculated the threshold by averaging the critical spacing values in correspondence of the last eight reversals.

\subsubsection{CSF stimuli}

We measured contrast sensitivity functions (CSF) by using sinusoidal gratings generated by a VSG2/3 graphics card with 12-bit luminance resolution. Gratings were displayed on a 17 -inch Philips Brilliance 107P CRT monitor with a refresh rate of $70 \mathrm{~Hz}$ and a spatial resolution of $1024 \times 768$ pixels. The stimuli were vertical gratings displayed on the whole screen area $(26 \times 20 \mathrm{deg})$. Contrast thresholds were measured with the method of Limits for each of the eight spatial frequencies tested (i.e., 0.1, 0.2, 0.4, 0.9, 2.0, 4.5, 10.2, and $20.4 \mathrm{cpd})$.

\subsection{Procedure}

\subsubsection{Pre- and post-training evaluation}

Participants performed a monocular and a binocular evaluation of central visual acuity (ETDRS; Landolt C), eccentric visual acuity, crowding, and finally the central contrast sensitivity function was measured. All of these tests were repeated after the training sessions. Moreover, participants were tested in the baseline conditions before and after the training.

\subsubsection{PL procedure}

A typical daily session consisted of 4 blocks, in which the target-to-flanker distance was varied starting from the higher distance (i.e., $8 \lambda$ ) and with the spatial frequency of the Gabor patches varying across daily sessions. Each treatment session was approximately 30 minutes in duration and was administrated twice a week. To probe specificity effects (Harris, Gilksberg, and Sagi, 2012), each of the two daily sessions was devoted to one of the two spatial frequencies, starting from the lower spatial frequency (i.e., $6 \mathrm{cpd}$ ). The PL was completed after six weeks.

\section{Results}

Overall, the results show weak facilitatory interactions in myopia, which are strengthened by PL. The training of facilitatory lateral interactions enhanced the contrast sensitivity function and increased visual acuity but did not reduce crowding. Moreover, the results show that these learning effects transferred to the untrained eye but not to either the horizontal collinear configuration of $6 \mathrm{cpd}$ or an orthogonal configuration (i.e., vertical triplet but with flankers orthogonal to the central target). In addition, the results did not show any transfer of learning to a collinear vertical configuration made up by Gabor patches with a lower spatial frequency (i.e., $1.5 \mathrm{cpd}$ ).

\subsection{Lateral interactions in myopia}

Figure 2 shows normalized thresholds (log units) as a function of the target-to-flanker distances for both normal sighted observers and myopic observers. Contrast thresholds, obtained in the collinear configuration of $6 \mathrm{cpd}$ at each target-to-flanker distance, were normalized for each individual observer by the baseline threshold obtained using an orthogonally flanked target with the same spatial frequency and target-to-flanker separation (i.e., $\log _{10}$ [collinear/orthogonal]).

The results of the repeated measures ANOVA performed to compare the two groups at the different levels of $\lambda$ showed a significant effect of Targetto-Flankers Separation $(F(3,36)=9.7, p=0.0001)$. Bonferroni corrected pairwise comparisons showed that mean normalized thresholds at $2 \lambda$ were significantly different to those at $3 \lambda$ and $4 \lambda$ Moreover, normalized thresholds at $3 \lambda$ significantly differed to those at $8 \lambda$. Neither the effect of the Group nor the interaction between Group and Target-to-Flankers separation resulted significant. However, Bonferroni corrected one-sample $t$-tests revealed that normal-

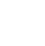

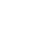

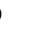




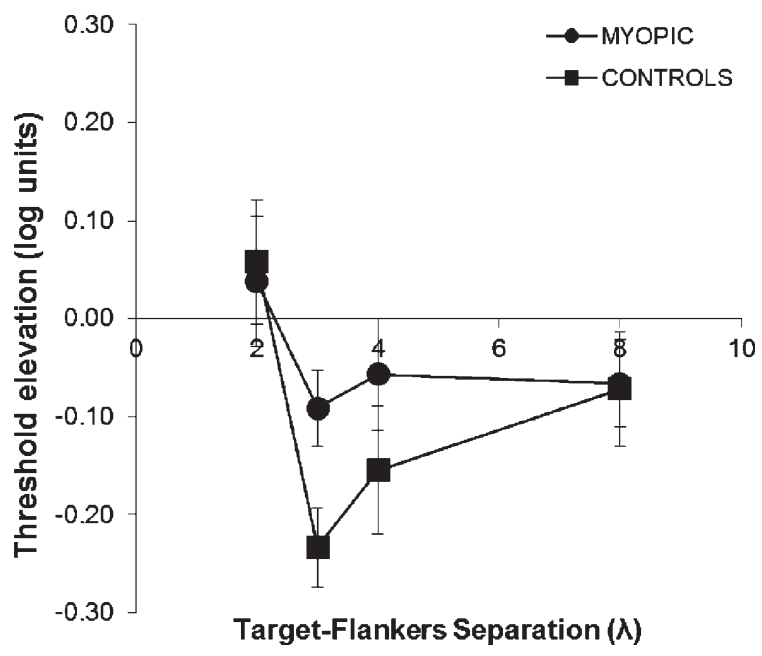

Fig. 2. Mean normalized thresholds (log units) for $6 \mathrm{cpd}$ target as a function of the target-to-flanker distances for both normal sighted observers and myopic observers. Thresholds in the collinear configurations were normalized by the baseline threshold of orthogonally flanked target. The mean normalized thresholds for target-to-flanker separations of $2 \lambda, 3 \lambda, 4 \lambda$ and $8 \lambda$ were equal to 0.037 (SEM: 0.066 ), -0.091 (SEM: 0.039), -0.056 (SEM: 0.057) and -0.066 (SEM: 0.044 ) for the myopic observers and were equal to 0.057 (SEM: $0.063),-0.23$ (SEM: 0.04), -0.15 (SEM: 0.065) and -0.07 (SEM: 0.058 ) for the control observers, respectively. Error bars \pm SEM.

ized thresholds obtained at $3 \lambda$ by the normal sighted observers $(t(6)=-5.7, p=0.001)$ were significantly different from zero. On the other hand, normalized thresholds obtained at $3 \lambda$ by the myopic observers $(t(6)=2.32, p=0.059)$ did not differ from zero. This result indicates facilitatory interactions at this targetto-flankers separation only for the sighted observers. At
$4 \lambda$ normalized thresholds of neither group significantly differed from zero (controls: $t(6)=-2.3, p=0.056$; myopic: $t(6)=0.992, p=0.36$ ). The amount of facilitation in the sighted observers was very similar to that reported in previous studies (Polat and Sagi, 1993, 1994a, 1994b; Shani and Sagi, 2005; Polat, 2009), whereas the amount of facilitation found in the myopic observers was significantly reduced. These results indicate a reduced strength of facilitatory lateral interaction in myopic observers.

\subsection{Perceptual learning}

The mean normalized thresholds obtained before and after the training by myopic observers are shown in Table 2 and Fig. 3. Table 2 also reports the contrast thresholds estimated in the pre- and posttraining sessions for the two spatial frequencies used and for the collinear and orthogonal conditions (i.e., baseline contrast thresholds). A three-way repeated measures ANOVA conducted on normalized thresholds before and after learning showed a significant effect of Learning $(F(1,6)=12.34, p=0.013)$, but neither a significant effect of the Spatial Frequency $(F(1,6)=0.01, p=0.97)$, nor a significant effect of the Target-to-Flanker Separation $(F(1,6)=2.3, p=0.14)$. Since we did not obtain a significant effect of the Spatial Frequency, data for the two spatial frequencies (i.e., 6 and $12 \mathrm{cpd}$ ) were collapsed to increase statistical power (see Fig. 3). The repeated measures ANOVA did not point out any significant interaction. Moreover, after training, normalized thresholds for the myopic observers were significantly lower
Table 2

Top panel: Mean contrast thresholds of the myopic observers obtained before (pre-) and after (post-) the training separately for 6 and 12 cpd, and for the collinear and orthogonal configurations. Bottom panel: Mean normalized thresholds of the myopic observers obtained in the preand post-training sessions separately for 6 and 12 cpd. SEM in brackets. * * Introduce "."

\begin{tabular}{|c|c|c|c|c|c|c|c|c|}
\hline & \multicolumn{8}{|c|}{ Contrast thresholds } \\
\hline & \multicolumn{2}{|c|}{ Pre-6cpd } & \multicolumn{2}{|c|}{ Post- $6 \mathrm{cpd}$} & \multicolumn{2}{|c|}{ Pre-12 cpd } & \multicolumn{2}{|c|}{ Post-12 cpd } \\
\hline & Coll. & Ortho. & Coll. & Ortho. & Coll. & Ortho. & Coll. & Ortho. \\
\hline $2 \lambda$ & $0.093(0.03)$ & $0.10(0.04)$ & $0.076(0.02)$ & $0.11(0.04)$ & $0.17(0.04)$ & $0.18(0.04)$ & $0.13(0.02)$ & $0.17(0.02)$ \\
\hline $3 \lambda$ & $0.097(0.04)$ & $0.11(0.04)$ & $0.080(0.02)$ & $0.12(0.03)$ & $0.18(0.06)$ & $0.19(0.04)$ & $0.12(0.02)$ & $0.19(0.03)$ \\
\hline $4 \lambda$ & $0.1(0.04)$ & $0.11(0.04)$ & $0.093(0.03)$ & $0.13(0.03)$ & $0.15(0.03)$ & $0.18(0.04)$ & $0.14(0.03)$ & $0.20(0.03)$ \\
\hline \multirow[t]{3}{*}{$8 \lambda$} & $0.11(0.04)$ & $0.12(0.03)$ & $0.10(0.04)$ & $0.12(0.03)$ & $0.17(0.03)$ & $0.18(0.04)$ & $0.18(0.02)$ & $0.20(0.03)$ \\
\hline & \multicolumn{8}{|c|}{ Normalized thresholds } \\
\hline & Pre-6cpd & & Post- 6 cpd & & Pre-12 cpd & & Post-12 cpd & \\
\hline $2 \lambda$ & $0.037(0.07)$ & & $-0.13(0.03)$ & & $-0.04(0.06)$ & & $-0.12(0.03)$ & \\
\hline $3 \lambda$ & $-0.091(0.04)$ & & $-0.23(0.07)$ & & $-0.07(0.07)$ & & $-0.18(0.05)$ & \\
\hline $4 \lambda$ & $-0.056(0.06)$ & & $-0.19(0.11)$ & & $-0.06(0.04)$ & & $-0.21(0.07)$ & \\
\hline $8 \lambda$ & $-0.066(0.04)$ & & $-0.08(0.07)$ & & $-0.01(0.05)$ & & $-0.09(0.03)$ & \\
\hline
\end{tabular}




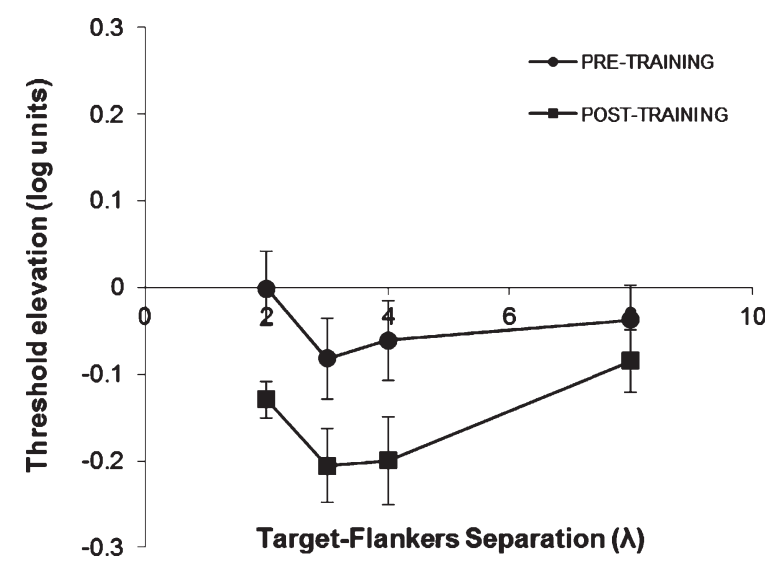

Fig. 3. Mean normalized thresholds (log units) obtained by the myopic observers before and after learning. Data relative to the 6 and $12 \mathrm{cpd}$ were collapsed (see Table 2 for actual values). Thresholds are shown as a function of target-to-flankers separation $(\lambda)$. Error bars \pm SEM.

than zero at $2 \lambda(t(6)=-6.2, p=0.001), 3 \lambda t(6)=-4.8$, $p=0.003)$ and $4 \lambda t(6)=-3.9, p=0.007)$, but not at $8 \lambda t(6)=-2.362, p=0.056)$. These results indicate that training the contrast detection of a collinearly flanked target resulted in a significant decrease of contrast thresholds. In addition, this improvement is specific for the trained stimulus, indeed learning effect did not transfer to a target of the same orientation flanked by orthogonal Gabors. These results suggest that perceptual learning improves visual performance specifically for the trained collinear stimulus, pointing to a general increase of facilitatory interactions in the visual cortex during training.

Since learning specificity is viewed as the main indicator of the level of processing at which learning takes place, we also tested the specificity of learning for spatial frequency, target-flankers global orientation (i.e., orientation of the triplet) and interocular transfer.

\subsection{Transfer of learning to lower spatial frequencies}

Figure 4 shows the contrast thresholds obtained by the myopic observers in the pre- and post-training sessions. We did not find any transfer of perceptual learning to the vertical collinear configuration with low spatial frequency Gabors (i.e., $1.5 \mathrm{cpd}$ ). A repeated measures ANOVA conducted on the contrast thresholds before and after learning did not report

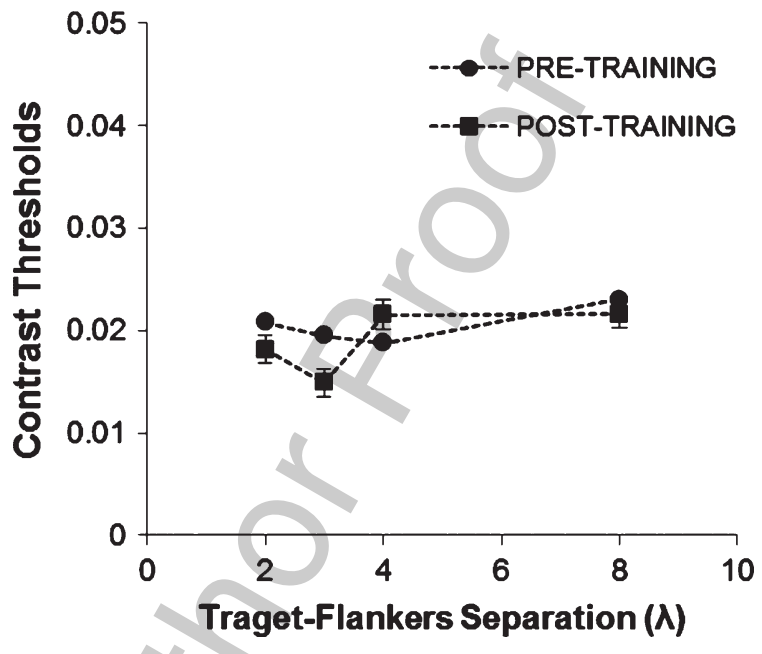

Fig. 4. Mean contrast thresholds (Michelson contrast) obtained in the untrained configuration of $1.5 \mathrm{cpd}$, as a function of targetto-flankers separation $(\lambda)$. The average thresholds for target-to flanker separations of $2 \lambda, 3 \lambda, 4 \lambda$ and $8 \lambda$ were respectively equal to 0.02 (SEM: 0.0013), 0.019 (SEM: 0.0013), 0.019 (SEM: 0.0014) and 0.023 (SEM: 0.0013 ) before the training and equal to 0.018 (SEM: 0.0014), 0.015 (SEM: 0.0013), 0.022 (SEM: 0.0023) and 0.022 (SEM: 0.002) after training higher spatial frequencies. Error bars \pm SEM. In some conditions error bars are smaller than the symbol size.

either a significant effect of Learning $(F(1,6)=1.6$, $p=0.252$ ), or an effect of the Target-to-Flanker Separation, though it was close to significance $(F(3,18)=4.3$, $p=0.051$ ), or a significant interaction between Learning and Target-to-Flankers Separation $(F(3,18)=2.77$, $p=0.102)$. Indeed we found that, at every targetto-flanker separation, contrast thresholds before and after the training did not differ significantly $(p>0.05)$. The reason could be that contrast thresholds for 1.5 cpd Gabors were too low before training to be further decreased by PL (see Fiorentini and Berardi, 1980 for similar results). However, it should be noted that despite contrast modulation thresholds were estimated using a 8-bit luminance resolution, on average, the contrast modulation thresholds obtained before and after the training across all the target-to-flanker separations were significantly higher than the minimum contrast modulation displayed by the screen (i.e., 0.0098 Michelson contrast). One sample $t$-test revealed that thresholds were not significantly lower than 0.02 Michelson contrast, with except at $3 \lambda$ after learning $(t(6)=-3.8, p=.01)$, and that in this condition the threshold value did not differ from 0.015 (Michelson contrast). In addition, to assess whether the Gabor function was represented faithfully at the 

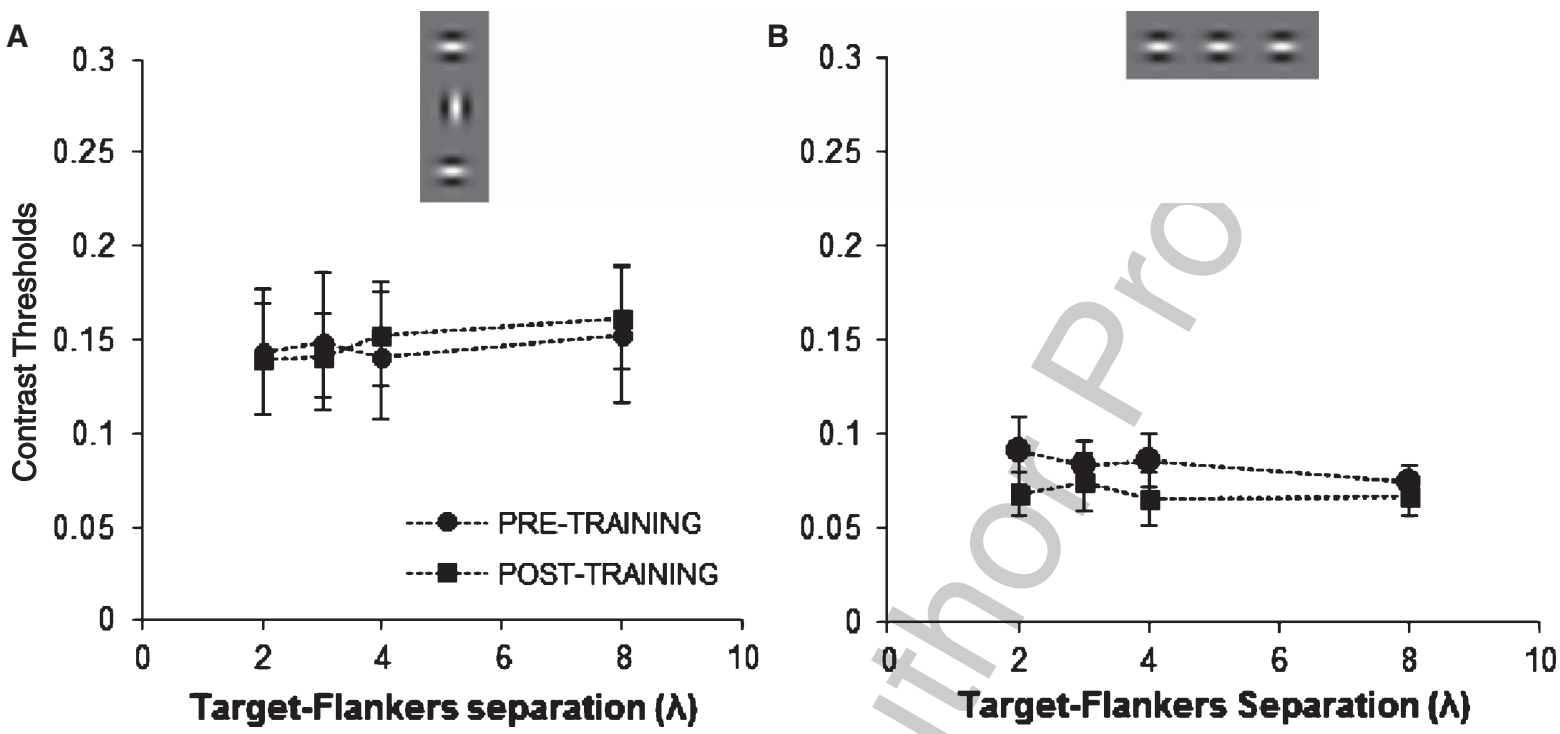

Fig. 5. Mean contrast thresholds (Michelson contrast) obtained in the following untrained configurations: (A) vertical configuration with vertical target and orthogonal flankers (data relative to the 6 and $12 \mathrm{cpd}$ were collapsed), and (B) horizontal collinear configuration only for 6 cpd condition. Mean contrast thresholds are represented as a function of the target-to-flankers separation $(\lambda)$. For target-to flanker separations of $2 \lambda, 3 *{ }^{\star}, 4 \lambda$ and $8 \lambda$, contrast thresholds in the orthogonal configuration are equal to 0.14 (SEM: 0.03), 0.15 (SEM: 0.03), 0.14 (SEM: 0.03) and 0.15 (SEM: 0.04 ) before the training and equal to 0.14 (SEM: 0.03), 0.14 (SEM: 0.02), 0.15 (SEM: 0.03) and 0.15 (SEM: 0.03) after training. In the horizontal configuration thresholds are equal to 0.09 (SEM: 0.02), 0.08 (SEM: 0.01), 0.08 (SEM: 0.01) and 0.07 (SEM: 0.01) before the training and equal to 0.07 (SEM: 0.01), 0.07 (SEM: 0.02), 0.06 (SEM: 0.01) and 0.07 (SEM: 0.01) after the training. Error bars \pm SEM.

minimum contrast modulation displayed by the screen (i.e., 0.0098 Michelson contrast), we performed a control experiment in which six naïve observers (normal sighted or corrected to normal) reported whether they perceive a number of stripes of the Gabor patch lower or equal/higher than three, by pressing one of two designated keys on a standard computer keyboard. A single Gabor patch was presented at the center of the screen. We used five contrast levels: 0.025, 0.02, 0.015, 0.01 , and 0.005 (Michelson contrast); each contrast level was repeated 15 times. The percentage of trials in which observers reported to perceive a number of stripes equal or higher than three was: $0.025: 100 \%$ [SEM: 0\%], 0.02:97.7\% [SEM: 2.2\%], 0.015:98.8\% [SEM: $1.1 \%$ ], 0.01:82.6\% [SEM: 15.7], 0.005:0\%). This suggest that at $\sim 0.01$ of contrast modulation the Gabor function was still visible. In sum, our results suggest that the learning effect is specific for the spatial frequency.

\subsection{Transfer of learning to different configurations}

A repeated measures ANOVA conducted on the contrast thresholds obtained before and after learn- ing with orthogonal configuration did not report any significant main effect or interaction: Learning $(F(1,6)=0.007, p=0.94)$, Target-to-Flanker Separation $(F(3,18)=0.95, p=0.42)$, Learning $\times$ Target-toFlankers Separation $(F(3,18)=0.77, p=0.46)$. Similarly, contrast thresholds obtained before and after learning with horizontal collinear configurations of 6 cpd Gabors (the $12 \mathrm{cpd}$ data were not included because only four observed were tested in this condition) did not report a significant effect of Learning $(F(1,6)=2.27, p=0.19)$, of the Target-to-Flanker Separation $(F(3,18)=0.43, p=0.61)$ and of the interaction between Learning and Target-to-Flankers Separation $(F(3,18)=0.76, p=0.47)$. These results indicate that there is not a transfer of learning to an orthogonal configuration (Fig. 5A) and to the horizontally oriented collinear configuration (Fig. 5B), presented in the same retinal position as the learning stimulus. Note however that three out of four subjects that performed the horizontal collinear condition with a spatial frequency of $12 \mathrm{cpd}$ showed transfer of learning, suggesting that training a vertical collinear configurations may transfer to horizontal collinear configuration, but only for high spatial frequencies. This second result was unexpected on the bases of previous results showing that, as task

* The symbol is wrong 478 479 480 481 482 483 484 485 487 

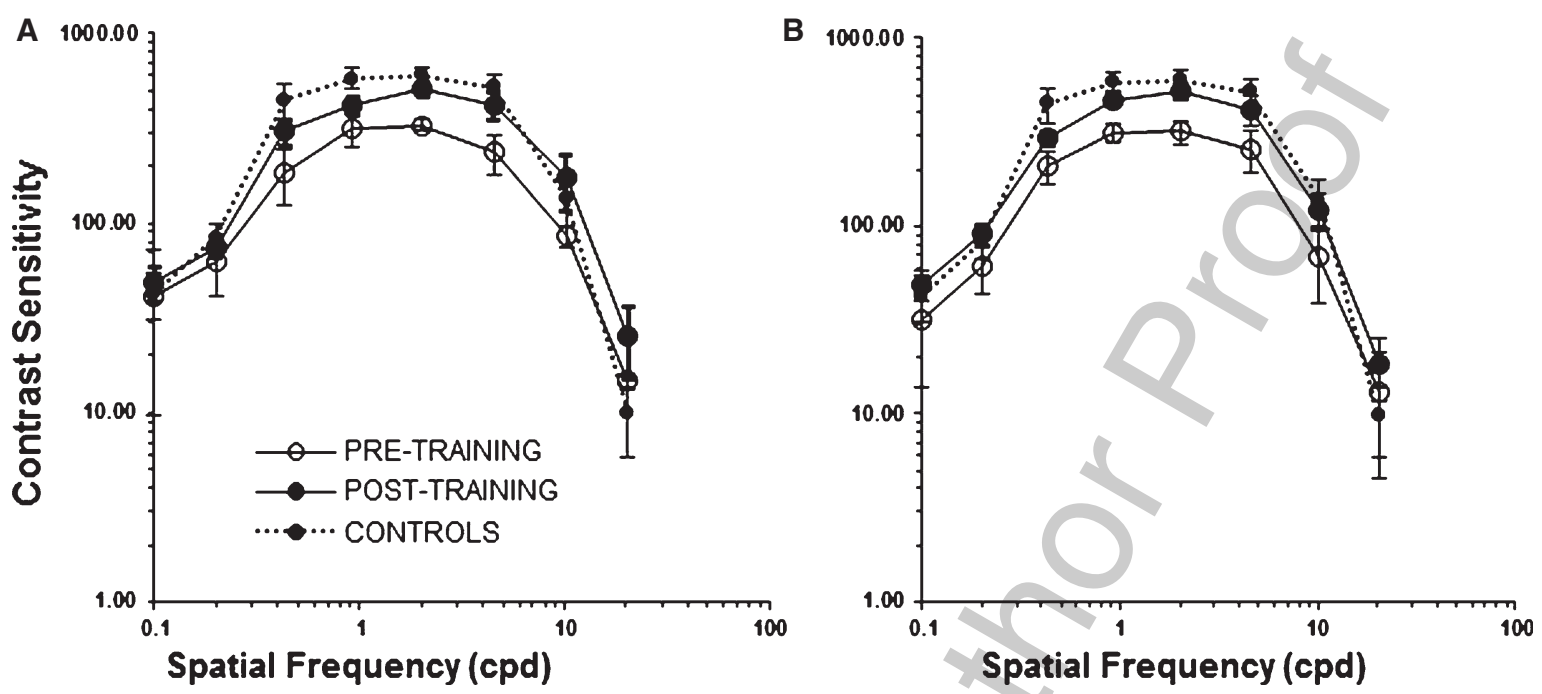

Fig. 6. Contrast sensitivity functions (CSFs) obtained in both the trained (A) and untrained (B) eye before and after training. The effect of training was significant at 2.04 cpd (trained: 330 [SEM: 47] vs. 512 [SEM: 45]; untrained: 325 [SEM: 47] vs. 513 [SEM: 44 ]), at 4.53 cpd (trained: 237 [SEM: 65] vs. 419 [SEM: 66]; untrained: 256 [SEM: 61] vs. 402 [SEM: 75]) and less consistently significant at 10.18 cpd (trained: 85 [SE: 30] vs. 172 [SE: 56]; untrained: 73 [SE: 32] vs. 114 [SE: 28]). Data are compared to those obtained by normal sighted observers. Error bars \pm SEM.

difficulty increases, learning becomes more specific with respect to orientation (Ahissar and Hochstein, 1996). However, a larger sample size is need to clarify whether the transfer to a horizontal collinear configuration depends on spatial frequency in the myopic eye.

The finding that transfer stimuli are immune to perceptual learning of vertical collinear configurations strongly suggests that the modulation of lateral interactions through perceptual learning is functionally specific. Ts'o and colleagues (1986) investigated the relationship between lateral connections and the functional architecture of V1; in particular, they showed that V1 neurons establish connections only with cells that have the same functional specificity (i.e., responsiveness to an iso-oriented line). Thus, our results are compatible with their findings.

\subsection{Transfer of learning to contrast sensitivity function (CSF)}

Contrast sensitivity for sinusoidal gratings was measured before and after training in order to derive CSFs (Fig. 6). Before training, the difference in contrast sensitivity between myopic and control observers was significant $(F(1,10)=12.1, p=0.006)$. The interaction between Group and Spatial Frequency was also significant $(F(1,10)=6.6, p=0.005)$. Bonferroni corrected pairwise comparisons reported a significant difference in contrast sensitivity between control and myopic observers before Learning at $0.4(p=0.015)$, $0.9(p=0.005), 2.0(p=0.005)$ and $4.5 \mathrm{cpd}(p=0.021)$. Training lateral interactions increased contrast sensitivity significantly in both the trained and untrained eye at $2.0 \mathrm{cpd}(p=0.002, p=0.02), 4.5 \mathrm{cpd}(p=0.005$, $p=0.0001)$ and less consistently at $10.18 \mathrm{cpd}(p=0.08$, $p=0.04$ ), indicating that Learning to detect flanked Gabor of 6 and $12 \mathrm{cpd}$ selectively transfers to gratings of similar (ranging from 4.5 to $10 \mathrm{cpd}$ ) and lower spatial frequencies ( $2 \mathrm{cpd}$ ). However, we did not report a corresponding improvement for Gabor patches in the orthogonal configuration at the trained spatial frequencies and for collinear Gabor patches of $1.5 \mathrm{cpd}$. Thus, there is transfer from flanked Gabors to wide gratings but not to non-collinear Gabors of the same or lower spatial frequency with respect to those trained. The transfer from Gabor patches to gratings cannot be due to an enhanced focused attention which would bring to an improvement of different spatial frequencies and configurations (Carrasco, Eckstein et al., 2000). More likely, the transfer from Gabor patches to grating may occur because training forces observers to simultaneously handle iso-oriented target and flankers so that it improves detection of the full screen grat- 

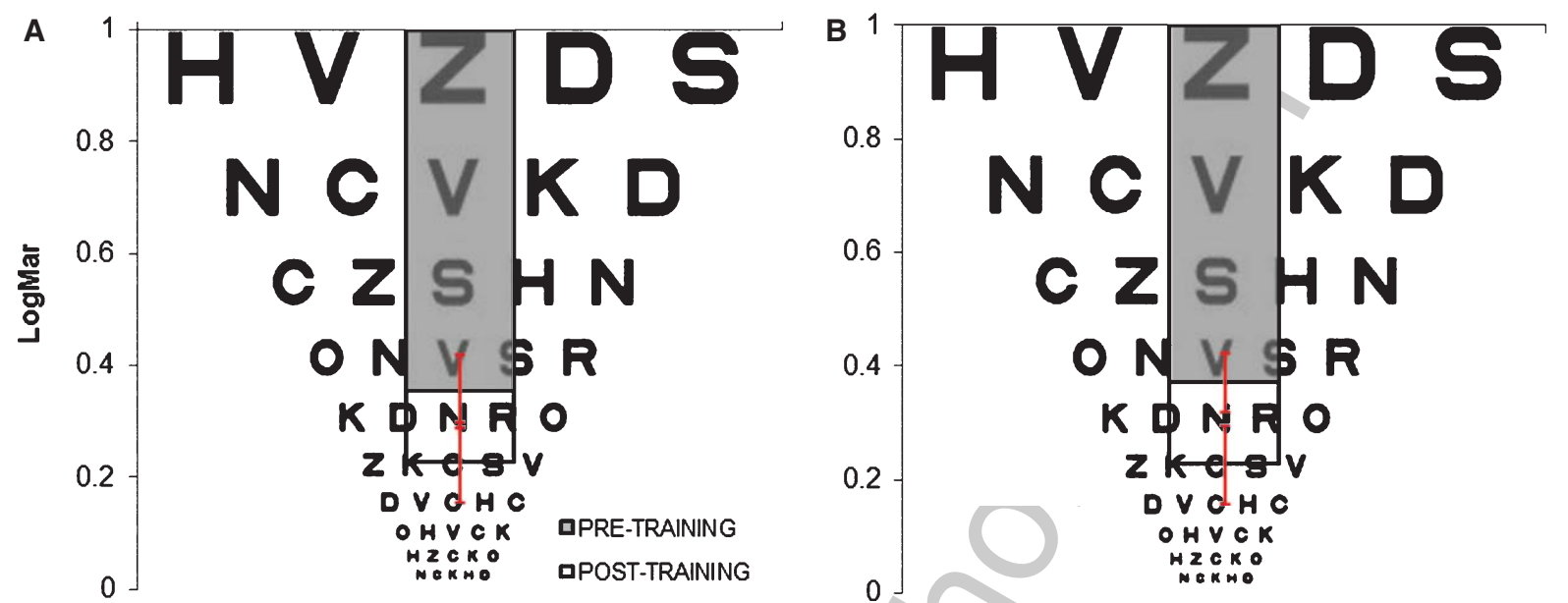

Fig. 7. Mean visual acuity (ETDRs) expressed in $\log M A R$, measured before (gray bars) and after training (transparent bars) for the trained eye (A: 0.36 [SEM: 0.06] and 0.23 [SEM: 0.07] logMAR) and the untrained eye (B: 0.37 [SEM: 0.05] and 0.23 [SEM: 0.06] logMAR). Error bars \pm SEM.

A

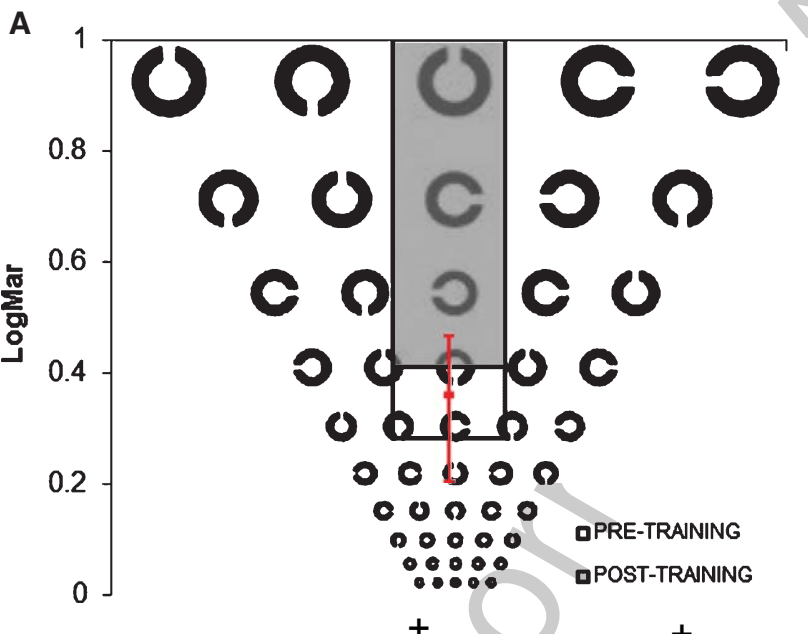

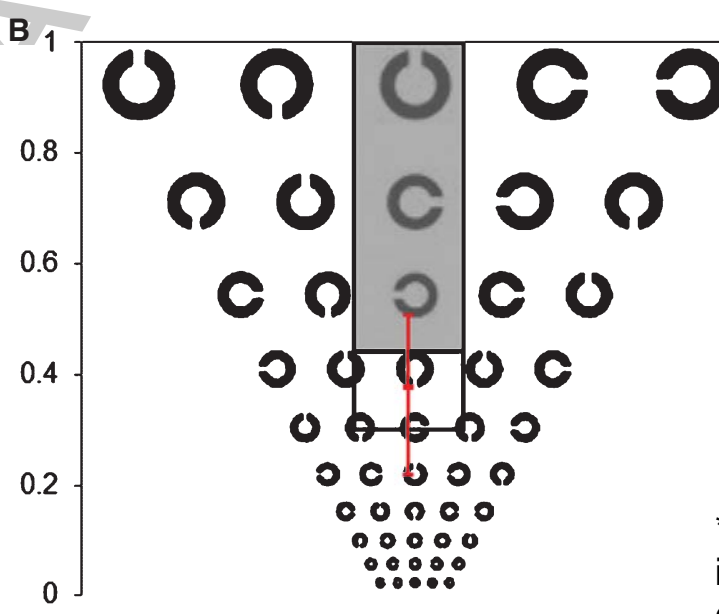

+ Pay close attention to the commas

Fig. 8. Mean visual acuity (Landolt C), expressed in $\operatorname{logMAR}$, measured before (grey bars) and after training (transparent bars) for the trained eye (A: 0.41 [SEM: 0.05] and 0.28 [SEM: 0.08] logMAR) and the untrained eye (B: 0.44 [SEM: 0.06] and 0.3 [SEM: 0.08] logMAR). Error bars \pm SEM.

ings used to measure CSF, which stimulates several iso-oriented visual channels (Robson and Graham, 1981).

\subsection{Transfer of learning to visual acuity}

Results (Figs. 7 and 8) show that the increase of facilitation after training significantly improved visual acuity both in the trained and untrained eye. Indeed, the result of the two-way repeated measures ANOVA on the ETDRS data (Fig. 7) pointed-out a significant effect of Learning $(F(1,6)=17.7, p=0.006)$ but not a significant effect of the Eye $(F(1,6)=0.02$, $p=0.89$ ), and of the interaction between Learning and Eye $(F(1,6)=0.07, p=0.80)$. Similar results were obtained in the Landolt-C task (Fig. 8) in which we obtained a significant effect of Learning $(F(1,6)=13.02, p=0.01)$, but not a significant effect of the Eye $(F(1,6)=0.3, p=0.6)$ nor a significant interaction between Learning and Eye $(F(1,6)=0.3, p=0.6)$. The average learning dependent improvement in visual acuity was 1.3 and $1.4 \log$ MAR lines in the trained and untrained eye, respectively. This effect is lower than that reported in previous studies (Durrie and McMinn,
+ Pay

close attention to the commas

\section{gray} instead of grey 

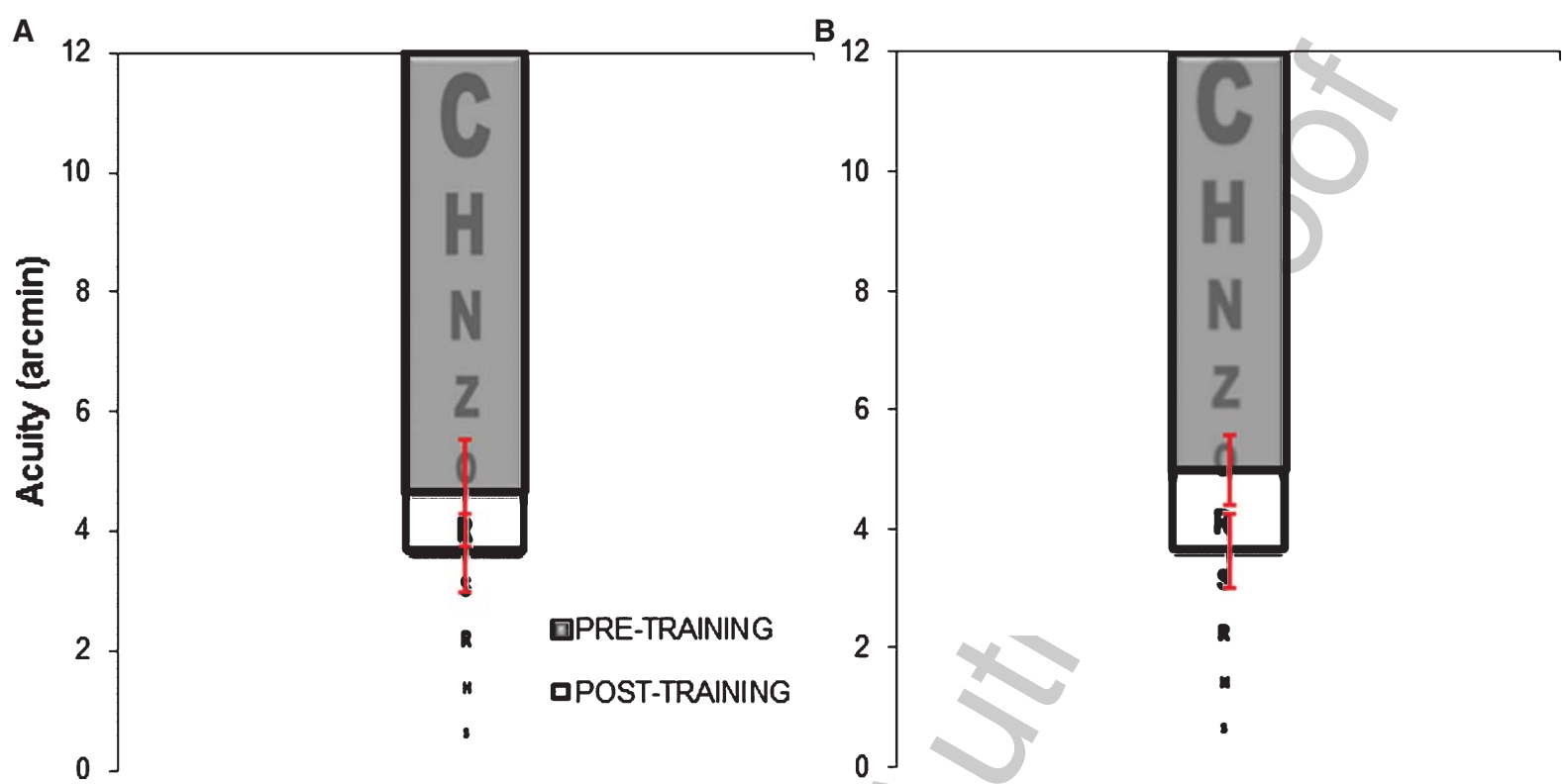

Fig. 9. Mean visual acuity, expressed in arcmin, obtained before (gray bars) and after training (transparent bars) for the trained eye (A: 4.7 [SE: 0.91] and 3.6 [SE: 0.67] arcmin) and the untrained eye (B: 5 [SE: 0.6] and 3.6 [SE: 0.62] arcmin). Stimuli were presented at 4 deg of eccentricity. Error bars \pm SEM.
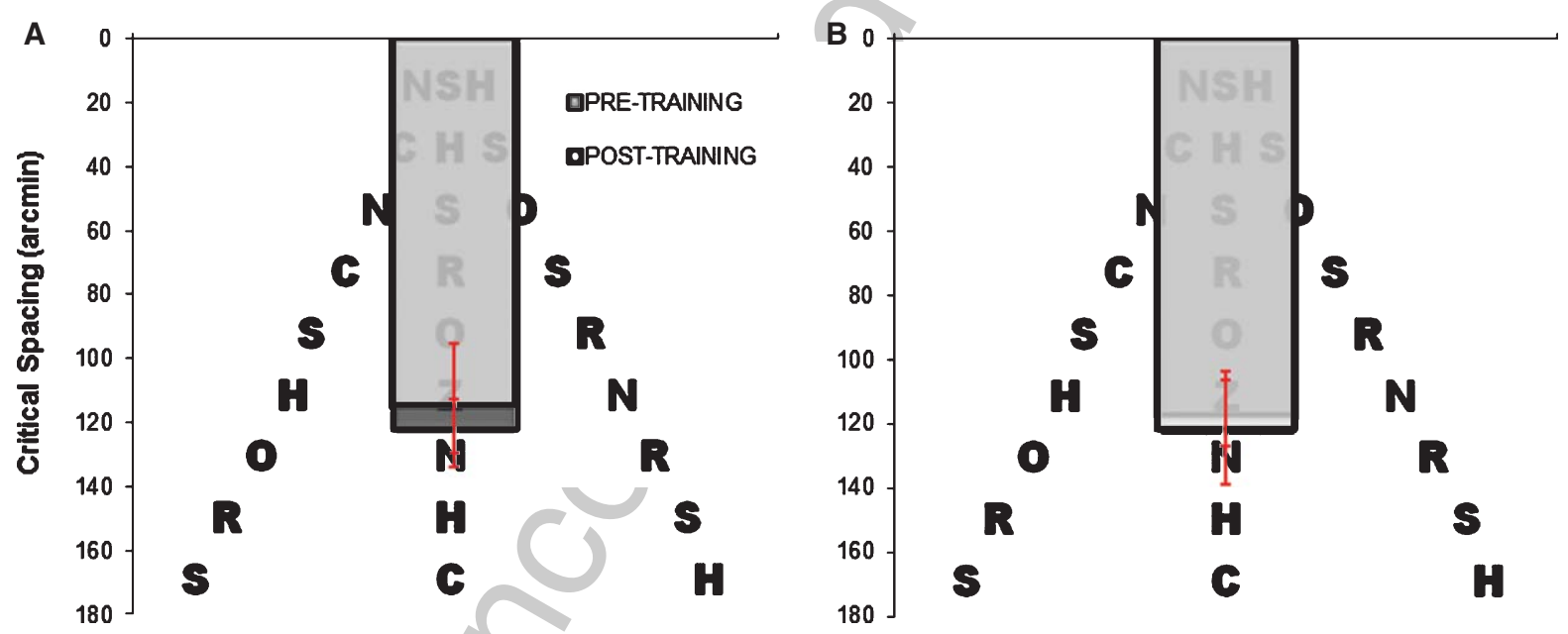

Fig. 10. Mean critical spacing (i.e., the target-to-flankers spacing at which the flankers did not reduce acuity), expressed in arcmin, obtained before (gray bars) and after training (transparent bars) for the trained eye (A: 121.1 [SEM: 8.5] and 114.4 [SEM: 19.1] arcmin) and the untrained eye (B: 116.9 [SEM: 10.1] and 121.2 [SEM: 17.7] arcmin). Stimuli were presented at 4 deg of eccentricity. Error bars \pm SEM.

2007; Tan and Fong, 2008), probably because we used only a vertically oriented training stimulus.

The transfer of learning with vertically oriented Gabors to complex stimuli such as Sloan letters and Landolt- $\mathrm{C}$ could be explained by assuming that these visual acuity tasks can be performed by detecting intensity changes (Westheimer, 2001), a task similar to the (trained) contrast detection.

\subsection{Transfer of learning to crowding}

Before Learning, eccentric visual acuity was significantly reduced in myopic with respect to control observers $(F(1,11)=8.9, p=0.012)$, whereas crowding was not $(F(1,11)=0.18, p=0.9)$. In myopic observers, training-dependent increased facilitation improved eccentric visual acuity (Fig. 9) but did not affect crowd- 
ing (Fig. 10). Indeed, a two-way ANOVA performed on the acuity data revealed a significant effect of Learning $(F(1,6)=11.6, p=0.014)$, but not a significant effect of Eye $(F(1,6)=2.5, p=0.17)$ nor a significant interaction between Learning and Eye $(F(1,6)=0.10, p=0.98)$. On average visual acuity after Learning increased by $24 \%$ for the trained eye and $28 \%$ for the untrained eye reaching the normal level $(F(1,11)=3.6, p=0.07)$, indicating that learning transferred to a more eccentric stimulus. On the other hand, a two-way repeated measures ANOVA performed on the crowding data did not report a significant effect of Learning $(F(1,6)=0.5$, $p=0.5)$, Eye $(F(1,6)=1.9, p=0.22)$ and of the interaction between Learning and Eye $(F(1,6)=2.3, p=0.18)$. This is expected since in normal vision critical spacing (i.e., the target-to-flankers distance at which the flankers no longer interfere with target identification) is equal to $\sim \frac{1}{2}$ the eccentricity (Bouma, 1970; Pelli, Palomares et al., 2004). That is, before Learning the crowding effect was within the range of normal vision in the myopic eye.

\section{Discussion}

In the present study we investigated the pattern of lateral interactions in observers with low myopia and its modulation by perceptual learning (PL). We used a contrast detection task of a central Gabor patch flanked above and below by two collinear high contrast Gabor patches. Observers had to report whether the central target was present in the first or second temporal interval of a two-interval display sequence (2IFC task). We assessed whether PL transferred to untrained stimuli and tasks, and whether it exhibits interocular transfer.

The first new result of the present study is a reduced strength of facilitatory lateral interactions in the myopic eye. This supports our suggestion that nonoptimal activation of high spatial frequency channels in individuals with myopia may also reduce the strength of facilitatory lateral interactions between them, in terms of the transmission of internal response via a cascade of local connections (Polat and Sagi, 1994b). This suggestion is indicative of maladaptive plasticity and it is also indirectly supported by the Hebbian rule, according to which mature visual cortical connections require temporal covariation, i.e., both preand postsynaptic activity within a defined time window (Karmarkar and Dan, 2006). It is also possible that cortical connections may not be mature at the onset of myopia so that they cannot become adult-like. To this purpose Kovacs (2000) has shown that longrange interactions span a shorter spatial range until the end of childhood. Indeed, if long-range lateral interactions are a late maturing function, they would be mostly affected by deficiency with a relatively late onset (Kovacs, 2000). However, in our myopic sample, the onset of myopia was relatively late during childhood, thus it is not clear whether this could have prevented a complete development of the pattern of lateral interactions. To address this issue, the comparison of the pattern of lateral interactions at different target-to-flankers distances $(\lambda)$ in myopic groups with different onset of myopia is needed.

Our second result is that training lateral interactions results into a significant facilitation induced by the flankers. In particular, we found lower contrast detection thresholds in the trained collinear configuration with respect to the untrained orthogonal configuration. This enhancement of facilitatory lateral interactions could result from learning dependent increments of the synaptic strength in response to repetitive coactivation of visual channels responding to target and flankers (Karmarkar and Dan, 2006). Co-activation of a network of visual channels could facilitate the recruitment of information within a large retinal area, thus explaining why Learning did not transfer to a vertically oriented target flanked by orthogonal Gabors but it enhanced contrast sensitivity function for large gratings. Indeed, we found that learning transfers to CSFs, and this occurs in both trained and untrained eye indicating that the mechanism involved in the collinear facilitation operates after the convergence of the visual input from the two eyes.

The third result is the evidence of specificity of learning effects. We found that training did not transfer to collinear configurations of low spatial frequency. The reason could be that CSF in myopic observers (Fig. 6) is slightly shifted towards low spatial frequencies for which there is little or no facilitation (i.e., contrast thresholds are slightly affected by target-to-flankers separation) (Fig. 4), possibly because of inefficient lateral interactions (Polat, 2009). Furthermore, the improvement of contrast sensitivity by training lateral interactions did not transfer orthogonal configurations of 6 and $12 \mathrm{cpd}$ and to collinear configurations of 6 cpd Gabors with different orientation of the triplet (i.e., horizontal; see Polat and Sagi, 1994b for similar results), although we found transfer of learning in three out of four subjects who were tested before 
and after the learning with a collinear horizontal configuration of $12 \mathrm{cpd}$. The specificity of learning in human adults for the low-level trained stimulus and for the task has been previously reported by a number of studies (Maniglia, Pavan et al., 2011; Bao, Yang et al., 2010; Gilbert, Sigman et al., 2001; Sagi and Tanne, 1994) as well as the selectivity to global configurations, both in normal sighted (Maniglia, Pavan et al., 2011) and in amblyopic observers (Levi and Polat, 1996; Levi, Polat et al., 1997; Li and Levi, 2004) and these results are consistent with the involvement of neural processes at the level of the primary visual cortex. It should be noted that the specificity phenomena we have reported may be the consequence of the procedure used that is likely to induce sensory adaptation. Indeed, Harris et al. (2012) using a texture discrimination task showed that location specificity is a consequence of sensory adaptation; that is, location specificity results from selective reduced sensitivity due to repeated stimulation. Observers practiced the texture discrimination task with the target presented at a fixed location within a background texture. To remove adaptation, the authors added task-irrelevant trials with the background texture oriented $45^{\circ}$ relative to the target's orientation. The results showed complete generalization to a new location when adaptation was removed, suggesting that adaptation interferes with invariant pattern-discrimination learning by inducing network-dependent changes in local visual representations.

The lack of transfer to the horizontal collinear configuration and to the orthogonal configuration tested with the same procedure, is indicative that learning cannot be accounted by changes in the optical system (e.g., refraction and accommodation). This is because changes of the refraction and accommodation would produce a non-specific improvement. Similarly, the transfer of learning to the non-trained eye indicates that its effect is neural and occurs after the information from the two eyes is combined (Fahle, 2004; Sagi, 2011). Moreover, Polat et al. (2012) showed that the learning dependent improvement of vision in the presbyopic eye is achieved without changing the optical characteristics of the eye.

In agreement with previous findings (Polat, 2009; Maniglia, Pavan et al., 2011; Polat, Ma-Naim et al., 2004; Durrie and McMinn, 2007; Tan and Fong, 2008) we found that the improvement achieved by perceptual learning of collinear configurations generalizes to complex tasks such as visual acuity with Landolt-
$\mathrm{C}$ and ETDRS, and such generalization occurs in the untrained eye in a more eccentric retinal position. One possible explanation for these transfer effects is that the improvement in contrast sensitivity may facilitate the performance of visual processing during the next stages of the visual cascade, where neurons respond to more complex input. To this purpose, a striking number of studies showed that PL can be transferred to the letter recognition task (VA) that relies on contrast detection (Chung, Legge et al., 2002; Chung, Mansfield et al., 1998; Legge, Pelli et al., 1985; Levi, Song et al., 2007; Majaj, Pelli et al., 2002; Patching and Jordan, 2005; Solomon and Pelli, 1994). Most of these studies indeed pointed out that letter recognition task is mediated by visual channels that rescale with letter size, such that when the channel's frequency and letter size are both expressed as retinal frequency $(\mathrm{c} / \mathrm{deg})$, a change in letter size will lead to a corresponding change in the underlying channel's frequency of the same magnitude. The change in terms of spatial frequency is usually described as a power function with an exponent of 1.0 (Solomon and Pelli, 1994).

There is a major question regarding the improvement of contrast sensitivity and visual acuity following $\mathrm{PL}$ in low myopia. The vision of myopic and presbyopic individuals is blurred without optical correction, and their corrected contrast sensitivity is reduced (Liou and Chiu, 2001). Despite blur had persisted for years, myopic subjects seem immune from effects due to adaptation to blur, that makes object to look sharper (Webster, Georgerson et al., 2002), improve visual resolution (Pesudovs and Brennan, 1993) and leading to unbiased perception (Yehezkel, Sagi et al., 2010). Instead, Perceptual Learning seems able to reduce blur. Such improvement could rely on some interaction between perceptual learning and visual adaptation. McGovern and colleagues (2012), for example, investigated the interaction between adaptation and perceptual learning in the motion domain. The results showed that, while adapting to motion, repeated practice of direction discrimination on the test pattern led to an improvement in direction discrimination performance that was higher after the training compared to the unadapted performance. Therefore, in the case of myopia it is possible that repeated practice in an adapted state to blur produces an improvement in contrast sensitivity. On the other hand, blur in myopia is long-lasting and the effects of Learning that we and other authors have shown (Durrie and McMinn, 2007; Tan and Fong, 2008) may also 
reflect learning-dependent modulation of a long-term maladaptive plasticity.

In conclusion, we showed that probing cortical interactions with a set of spatial frequencies and target-to-flankers separations can modulate the spatial interactions in the visual field of myopic adult observers. A relevant question is whether these results could have an important implication in the treatment of myopic individuals, improving their visual functions in everyday life. To this purpose learning effects should be retained. Although we did not collect follow-up data, this issue was addressed by other authors that used the same Learning paradigm in myopia. For example, Tan and Fong (2008) found a PL effect retained up to 12 months.

\section{Acknowledgments}

This work was supported by the University of Padova and the CARIPARO. Author AP was supported by the Alexander von Humboldt Foundations. ${ }^{*}$

* Should be: Alexander von Humboldt Foundation

\section{References}

Adini, Y., Sagi, D., \& Tsodyks, M. (2002). Context-enabled learning in the human visual system. Nature, 415, 790-793.

Ahissar, M., \& Hochstein, S. (1996). Learning pop-out detection: Specificities to stimulus characteristics. Vis Res, 36, 3487-3500.

Ball, K., \& Sekuler, R.A. (1982). Specific and enduring improvement in visual motion discrimination. Science, 218, 697-698.

Ball, K., \& Sekuler, R. (1987). Direction-specific improvement in motion discrimination. Vis Res, 27, 953-965.

Ball, K., Sekuler, R., \& Machamer, J. (1983). Detection and identification of moving targets. Vis Res, 23, 229-238.

Bao, M., Yang, L., Rios, C., He, B., \& Engel, S.A. (2010). Perceptual learning increases the strength of the earliest signals in visual cortex. J Neurosci, 30, 15080-15084.

Bouma, H. (1970). Interaction effects in parafoveal letter recognition. Nature, 226, 177-178.

Brainard, D.H. (1997). The psychophysics toolbox. Spatial Vision, $10,433-436$.

Campana, G., \& Casco, C. (2003). Learning in combined-feature search: Specificity to orientation. Percept Psychophys, 65, 1197-1207.

Carrasco, M., Eckstein, M., \& Penpeci-Talgar, C. (2000). Spatial covert attention increases contrast sensitivity across the CSF: Support for signal enhancement. Vis Res, 40, 1203-1215.

Casco, C., \& Campana, G. (1999). Spatial interactions in simple and combined-feature visual search. Spatial Vision, 12, 467-683.

\section{* Should be: Neurosci Lett}

Casco, C., Campana, G., Grieco, A., \& Fuggetta, G. (2004) Perceptual learning modulates electrophysiological and psychophysical response to visual texture segmentation in humans. * Neurosci Lettwi 371, 18-23.

Casco, C., Caputo, G., \& Grieco, A. (2001). Discrimination of an orientation difference in dynamic textures. Vis Res, 41, 275-284

Chung, S.T., Legge, G.E., \& Tjan, B.S. (2002). Spatial-frequency characteristics of letter identification in central and peripheral vision. Vis Res, 42, 2137-2152.

Chung, S.T., Mansfield, J.S., \& Legge, G.E. (1998). Psychophysics of reading. XVIII. The effect of print size on reading speed in normal peripheral vision. Vis Res, 38, 2949-2962.

Dorais, A., \& Sagi, D. (1997). Contrast masking effects change with practice. Vis Res, 37, 1725-1733.

Dosher, B.A., \& Lu, Z.L. (2005). Perceptual learning in clear displays optimizes perceptual expertise: Learning the limiting process. P Natl Acad Sci USA, 102, 5286-5290.

Durrie, D., \& McMinn, P.S. (2007). Computer-based primary visual cortex training for treatment of low myopia and early presbyopia. T Am Ophthal Soc, 105, 132-138.

Fahle, M. (2004). Perceptual learning: A case for early selection. $J$ Vision, 4, 879-890.

Fiorentini, A., \& Berardi, N. (1981). Learning in grating waveform discrimination: Specificity for orientation and spatial frequency. Vis Res, 21, 1149-1158.

Fiorentini, A., \& Berardi, N. (1980). Perceptual learning specific for orientation and spatial frequency. Nature, 287, 43-44.

Geisler, W.S., \& Albrecht, D.G. (1997). Visual cortex neurons in monkeys and cats: Detection, discrimination, and identification. Visual Neurosci, 14, 897-919.

Gilbert, C.D., Li, W., \& Piech, V. (2009). Perceptual learning and adult cortical plasticity. J Physiol, 587, 2743-2751.

Gilbert, C.D., Sigman, M., \& Crist, R.E. (2001). The neural basis of perceptual learning. Neuron, 31, 681-697.

Gilbert, C.D., \& Wiesel, T.N. (1983). Clustered intrinsic connections in cat visual cortex. $J$ Neurosci, 3, 1116-1133.

Grieco, A., Casco, C., \& Roncato, S. (2006). Texture segregation on the basis of contrast polarity of odd-symmetric filters. Vis Res, $46,3526-3536$

Harris, H., Gliksberg, M., \& Sagi, D. (2012). Generalized perceptual learning in the absence of sensory adaptation. Curr Biol, 22 , 1813-1817.

Karmarkar, U.R., \& Dan, Y. (2006). Experience-dependent plasticity in adult visual cortex. Neuron, 52, 577-585.

Karni, A., \& Sagi, D. (1991). Where practice makes perfect in texture discrimination: Evidence for primary visual cortex plasticity. $P$ Natl Acad Sci USA, 88, 4966-4970.

Kovács, I. (2000). Human development of perceptual organization. Vision Res, 40, 1301-1310.

Legge, G.E., Pelli, D.G., Rubin, G.S., \& Schleske, M.M. (1985). Psychophysics of reading-I. Normal vision. Vis Res, 25, 239252.

Levi, D.M., \& Polat, U. (1996). Neural plasticity in adults with amblyopia. P Natl Acad Sci USA, 93, 6830-6834.

\section{* Should be: Vis Res}


Levi, D.M., Polat, U., \& Hu, Y.S. (1997). Improvement in Vernier acuity in adults with amblyopia Practice makes better. Invest Ophth Vis Sci, 38, 1493-1510.

Levi, D.M., Song, S., \& Pelli, D.G. (2007). Amblyopic reading is crowded. J Vision, 7(2), 21, 1-17.

Levitt, H. (1971). Transformed up-down methods in psychoacoustics. J Acoust Soc Am, 49(Suppl 2), 467.

Li, R.W., \& Levi, D.M. (2004). Characterizing the mechanisms of improvement for position discrimination in adult amblyopia. $J$ Vision, 7(6), 476-487.

Liou, S.W., \& Chiu, C.J. (2001). Myopia and contrast sensitivity function. Curr Eye Res, 22, 81-84.

Maehara, G., \& Goryo, K. (2007). Perceptual learning in monocular pattern masking: Experiments and explanations by the twin summation gain control model of contrast processing. Percept Psychophys, 69, 1009-1021.

Majaj, N.J., Pelli, D.G., Kurshan, P., \& Palomares, M. (2002). The role of spatial frequency channels in letter identification. Vis Res, 42, 1165-1184.

Maniglia, M., Pavan, A., Cuturi, L.F., Campana, G., Sato, G., \& Casco, C. (2011). Reducing crowding by weakening inhibitory lateral interactions in the periphery with perceptual learning. PLoS One, 6(10), e25568.

Mayer, M.J. (1983). Practice improves adults' sensitivity to diagonals. Vis Res, 23, 547-550.

McGovern, D., Roach, N.W., \& Webb, B.S. (2012). Perceptual learning reconfigures the effects of visual adaptation. J Neurosci, 32, 13621-13629.

McKee, S.P., \& Westheimer, G. (1978). Improvement in vernier acuity with practice. Percept Psychophys, 24, 258-262.

Patching, G.R., \& Jordan, T.R. (2005). Spatial frequency sensitivity differences between adults of good and poor reading ability. Invest Ophth Vis Sci, 46, 2219-2224.

Pelli, D.G. (1997). The VideoToolbox software for visual psychophysics: Transforming numbers into movies. Spatial Vision, 10, 437-442.

Pelli, D.G., Palomares, M., \& Majaj, N.J. (2004). Crowding is unlike ordinary masking: Distinguishing feature integration from detection. Jommal of Vision, 4(12), 1136-1169.

Pesudovs, K., \& Brennan, N.A. (1993). Decreased uncorrected vision after a period of distance fixation with spectacle wear. Optom Vis Sci, 70, 528-531.

Poggio, T., Fahle, M., \& Edelman, S. (1992). Fast perceptual learning in visual hyperacuity. Science, 256, 1018-1021.

Polat, U. (2009). Making perceptual learning practical to improve visual functions. Vis Res, 49, 2566-2573.

\section{* Should be: $\mathrm{J}$ vision}

Polat, U., \& Sagi, D. (1993). Lateral interactions between spatial channels: Suppression and facilitation revealed by lateral masking experiments. Vis Res, 33, 993-999.

Polat, U., \& Sagi, D. (1994a). The architecture of perceptual spatial interactions. Vis Res, 34, 73-78.

Polat, U., \& Sagi, D. (1994b). Spatial interactions in human vision From near to far via experience-dependent cascades of connections. P Natl Acad Sci USA, 91, 1206-1209.

Polat, U., Ma-Naim, T., Belkin, M., \& Sagi, D. (2004). Improving vision in adult amblyopia by perceptual learning. P Natl Acad Sci USA, 101, 6692-6697.

Polat, U., Schor, C., Tong, J.L., Zomet, A., Lev, M., Yehezkel, O. Sterkin, A., \& Levi, D.L. (2012). Training the brain to overcome the effect of aging on the human eye. Scientific Reports, 2, 278.

Robson, J.G., \& Graham, N. (1981). Probability summation and regional variation in contrast sensitivity across the visual field. Vis Res, 21, 409-418.

Rockland, K.S., \& Lund, J.S. (1983). Intrinsic laminar lattice connections in primate visual cortex. J Comp Neurol, 216, 303-318.

Sagi, D., \& Tanne, D. (1994). Perceptual learning: Learning to see. Curr Opin Neurobiol, 4, 195-199.

Schoups, A., Vogels, R., Qian, N., \& Orban, G. (2001). Practising orientation identification improves orientation coding in $\mathrm{V} 1$ neurons. Nature, 412, 549-553.

Shani, R., \& Sagi, D. (2005). Eccentricity effects on lateral interactions. Vis Res, 45, 2009-2024.

Solomon, J.A., \& Pelli, D.G. (1994). The visual filter mediating letter identification. Nature, 369, 395-397.

Sowden, P.T., Rose, D., \& Davies, I.R. (2002). Perceptual learning of luminance contrast detection: Specific for spatial frequency and retinal location but not orientation. Vis Res, 42, 1249-1258.

Tan, D.T., \& Fong, A. (2008). Efficacy of neural vision therapy to enhance contrast sensitivity function and visual acuity in low myopia. J Cataract Refr Surg, 34, 570-577.

Ts'o, D.Y., Gilbert, C.D., \& Wiesel, T.N. (1986). Relationships between horizontal interactions and functional architecture in cat striate cortex as revealed by cross-correlation analysis. $J$ Neurosci, 6, 1160-1170.

Webster, M.A., Georgeson, M.A., \& Webster, S.M. (2002). Neural adjustments to image blur. Nat Neurosci, 5, 839-840.

Westheimer, G. (2001). Is peripheral visual acuity susceptible to perceptual learning in the adult? Vis Res, 41, 47-52.

Yehezkel, O., Sagi, D., Sterkin, A., Belkin, M., \& Polat, U. (2012). Learning to adapt: Dynamics of readaptation to geometrical * distortions. Vision Res, 50, 1550-1558.

\section{* Should be: Vis Res}

